\title{
Individual differences in the capacity limitations of visuospatial short-term memory: Research on sighted and totally congenitally blind people
}

\author{
CESARE CORNOLDI, ALBERTO CORTESI, and DARIA PRETI \\ University of Padua, Padua, Italy
}

\begin{abstract}
The study of visuospatial imagery processes in totally congenitally blind people makes it possi. ble to understand the specific contribution of visual experience for imagery processes. We argue that blind people may have visuospatial imagery processes, but they suffer from some capacity limitations. Similar, although smaller, limitations and individual differences may be found in sighted people. Visuospatial imagery capacity was explored by asking people to follow an imaginary pathway through either two- or three-dimensional matrices of different complexity. The blind appear to use specific visuospatial processes in this task (Experiments 2 and 3), but they have difficulty with three-dimensional matrices; sighted people have no such difficulty with threedimensional matrices (Experiment 1). On the other hand, when a three-dimensional pattern exceeded sighted capacity, the blind and sighted showed similar patterns of errors. Subsequent analyses suggested that both visuospatial processes and verbal mediation were used.
\end{abstract}

A large part of the literature on visuospatial imagery processes in blind people has focused on the consequences of the absence of visual experience in cognitive processes typically attributed to imagery. The most obvious assumption has been that, if visuospatial imagery is essentially the by-product of visual experience, people who have never had any visual experience (e.g., the totally congenitally blind) should fail or at least behave differently from the sighted in tasks that presumably require the use of visuospatial imagery. Relevant evidence is contradictory, however. On the one hand, blind and sighted people show identical performance in a variety of cognitive tasks, such as mental rotation (Marmor \& Zaback, 1976), mental scanning (Kerr, 1983), memory for high imagery stimuli (Craig, 1973), and memory following visual imagery instructions (Jonides, Kahn, \& Rozin, 1975). As Zimler and Keenan (1983) suggest, an obvious conclusion is that these tasks do not require (or do not necessarily require) the use of imagery. On the other hand, Kerr (1983) argues that blind people may have visual images. De Beni and Cornoldi (1988) agree and contend that the integrity of other sensory modalities and of the visuospatial cortical area may generate visual images whose sources of information can be perceptual, but not visual. They also argue that the main purpose of research on visuospatial imagery in the blind should focus on its specific limitations as an opportunity to find the conditions under which visual

We are grateful to $S$. Feduzi, who helped in collecting the data, and to M. Heller, N. Kerr, and M. Marschark for their useful suggestions. The research was partially supported by a CNR grant to the first author. Requests for reprints should be sent to C. Cornoldi. Dipartimento di Psicologia Generale, Università Degli Studi di Padova, Piazza Capitaniato 3. 35139 Padova, Italy. experience is critical for visuospatial imagery. They further suggest that a specific limitation may be found in visuospatial buffer when it is highly loaded.

Research on visuospatial imagery has focused on the role of visuospatial short-term memory in the generation and maintenance of visual images (Baddeley, 1986; Kosslyn, 1980 ). In a recent review of the literature, Logie (1990) examined the convergence of different approaches, with particular reference to Kosslyn's "visual buffer" and to Baddeley's "visuospatial sketch pad." These concepts, particularly that of the visuospatial sketch pad, have clear implications for the capacity of visuospatial short-term memory

Baddeley's (1986) working-memory model proposes a central executive system and different slave systems related to different sensory modalities. Verbal and visuospatial components of short-term memory are considered independent and parallel in some respects. Baddeley suggests that the same problems analyzed for the verbal component, the articulatory loop, should be considered for the visuospatial component, the visuospatial sketch pad or scratch pad, but admits relevant research is still sketchy. One deficiency is the capacity of the visuospatial component (see Logie, 1989, for a short review).

Kerr (1987) studied the capacity of people's visual working memory while processing either two- or threedimensional visuospatial information of different levels of complexity. Subjects were required to imagine square matrices or cubes formed by an increasing number of units and to follow a mental pathway to its final position. The result was a new measure for testing visuospatial workingmemory capacity.

In our opinion, this measure has a number of advantages. It is not restricted to two-dimensional shapes, and 
it allows the exploration of the visuospatial sketch pad, reasonably unrestricted by language or by other strong constraints. The procedure also permits the measurement of short-term memory capacity while information undergoes some processing. The latter advantage is important because tasks requiring the processing of maintained information differ from simple tests of recall of configurations (e.g., Phillips \& Christie, 1977), in that some cognitive resources (perhaps the activity of a central executive, Baddeley, 1986) are required by the task carried out on the maintained information. This reasoning suggests that the task involving information maintained in short-term memory should be simple and easily available to each tested subject. In this respect, the task of following a mental pathway appears simple, and its success seems fundamentally due to the possibility of maintaining in memory the configuration along which the subject has to move mentally.

Kerr's (1987) main result was that the capacity limitations of visuospatial short-term memory are defined by the number of units per dimension, not by the number of represented units. Subjects easily processed three units independently of whether two dimensions (as in a $3 \times 3$ matrix for a total of nine units) or three dimensions (as in a $3 \times 3 \times 3$ matrix for a total of 27 units) were involved. When the memory load exceeded this limitation, the number of units became relevant; at an even number of units, three-dimensional shapes were easier to process; at an even number of units per dimension, two-dimensional shapes are easier.

Kerr's (1987) results are partially counterintuitive, given the fact that three-dimensional patterns could be more difficult not only because they involve a larger number of units, but also because they have some concealed units, which, according to some authors (e.g., Keenan \& Moore, 1979), should be difficult for imagery. For example, in a cube made up of 27 small cubes and placed on a desk, the central cube is not visible from any perspective and the six lower back cubes cannot be seen from the spectator's point of view. The fact that subjects did not have difficulty with them confirms Neisser and Kerr's (1973) data that imagery can process parts that are invisible from a perceptual point of view.

The main purpose of the current research was to explore the generality and the specifications required for Kerr's (1987) conclusions. We hypothesized that, with verbal short-term memory capacity, individual differences would appear in visuospatial short-term memory. We first examined this issue from the point of view of the totally congenitally blind, because research on these subjects seems particularly promising with respect to the problem of imagery limitations. De Beni and Cornoldi (1988) found that totally congenitally blind subjects were able to take advantage of instructions to create interactive images as effectively as sighted subjects when the load was small (one or two items) but failed when the memory load was increased. Cornoldi, De Beni, Roncari, and Romano (1989) then found that blind subjects' deficit was specific to imaginal processing and was absent when verbal strategies were required. They argued that the blind are able to create visual images, but with specific capacity limitations. The current research used Kerr's (1987) adaptation of Attneave and Curlee's (1983) paradigm to test the hypothesis that totally congenitally blind people, too, may create and use visuospatial images, but they have limitations. Furthermore, we wanted to explore whether the number of dimensions had the same effect on blind as on sighted people.

We hypothesized that visuospatial short-term memory of the blind would be more limited by the number of dimensions than would comparable performance by the sighted. Although this problem has not been directly examined in the literature, some evidence suggests that blind people have difficulty with depth. For example, Millar (1975) observed that blind children had more difficulty than did sighted children in reproducing the more distant position of a five-sided open maze. Similar difficulties in the reproduction of distant positions were also observed with adult blind subjects (Shagan \& Goodnow, 1973). However, processing a third dimension seems to more directly involve the spatial components rather than the visual components of imagery, and the blind, if they are better in spatial imagery than in visual imagery (Logie, 1990), should not be particularly disadvantaged.

A final purpose of the present research was to examine our hypothesis that individual differences represent a general problem, not limited to exceptional cases, for visuospatial short-term memory capacity. Our hypothesis was not only a consequence of a theoretical reflection on the blind, but it was also based on the classical data on the psychological differentiation in visuospatial abilities, found in a variety of tests, such as the Differential Aptitudes Test and the Minnesota Paper Form Board. Because highly discriminative visuospatial tests make large use of three-dimensional shapes, it is possible that only subjects with a high visuospatial ability are unaffected by the shift from two to three dimensions. This issue was not considered by Kerr (1987), whose experiments did not examine visuospatial ability, and could explain some of the small differences she found between experiments (e.g., with the critical $3 \times 3 \times 3$ matrix).

Finally, the current study examined the hypothesis that visuospatial short-term memory capacity depends largely on individual differences and that only some subjects with good visuospatial ability would cope with three dimensions as readily as with two. We assumed that the totally congenitally blind have more severe capacity limitations on their visuospatial imagery than do the sighted. This capacity difference should be more evident for threedimensional matrices than for two-dimensional matrices.

The use of blind subjects required slight modifications of the methodology used by Kerr (1987). Specifically, we blindfolded the sighted subjects. Furthermore, the tactile nature of two-dimensional patterns was made more explorable by using cubes rather than plane matrices. Finally, given the assumption that both number of units 
(cubes) and number of dimensions may be critical, we selected three two-dimensional patterns having three different levels of complexity $(3 \times 3,4 \times 4$, and $8 \times 8)$ and three three-dimensional patterns $(2 \times 2 \times 2,3 \times 3 \times 3$, and $4 \times 4 \times 4$ ) partly comparable to the two-dimensional patterns in the units for side and partly comparable to the overall number of units. In all other respects, the procedure resembled that of Kerr's (1987) Experiment 1.

\section{EXPERIMENT 1}

\section{Method}

Subjects. Twenty subjects ( 6 males and 14 females), classified as totally and congenitally blind, aged between 15 and 60 years (mean age $=32$ years, 11 months), took part in Experiment 1 . The subjects' blindness never resulted from diseases of the central nervous system (see Appendix A). Following the Italian classification tradition, we considered people to be totally blind when they could not perceive shapes and positions of the objects and to be congenitally blind when the deficit was present at birth or appeared in the first months of life. Some subjects, who had a minimal visual residual for light intensity or had developed the handicap in the first year of life, also were classified as totally congenitally blind, because they were thought to lack enough visual experience to have a visual representation of objects. Most subjects had a school diploma, obtained after having attended normal schools from 10 to 13 years. Controls were 20 sighted subjects matched for age, sex, and school level. The school level matching referred not only to the same years of schooling, but also to the kind of school (attended by both blind and sighted people), ensuring that similar experiences were offered to the two groups of subjects. The two groups had similar verbal short-term memory abilities on the WAIS span test. Mean performances of the blind group were 7.00 at the forward span and 5.50 at the backward span; mean performances of the control group were 7.25 and 5.70 , respectively. The performances were not significantly different using two $t$ tests.

Materials. Wooden cubes measuring $4 \mathrm{~cm}$ in each direction were used to prepare the following two- and three-dimensional patterns: $3 \times 3,4 \times 4$, and $8 \times 8$ (two-dimensional), $2 \times 2 \times 2,3 \times 3 \times 3$, and $4 \times 4 \times 4$ (three-dimensional). The patterns resembled those of Kerr's (1987) Experiment 3: The $2 \times 2 \times 2$ pattern was comparable for number of cubes to the $3 \times 3$ pattern; the $4 \times 4$ pattern was comparable in complexity of each side to the $4 \times 4 \times 4$ pattern.

Ten pathways were generated for each cube starting either from the cube close to the subject (at his or her left and at the highest layer for the three-dimensional shapes) or from a cube far from the subject (at his or her left and at the lowest layer for the threedimensional shapes): the first six trials (two practice trials and four experimental trials) started from one point, and the other four from the other point. Each pathway included a series of seven statements of direction, read through a tape recorder at a 2 -sec rate. Directions were left-right, forward-backward, and top-bottom (the last directions were used only for the vertical direction of the threedimensional patterns). The pathway never involved more than two movements in the same direction.

Procedure. The subjects were tested individually, seated close to the pattern. The sighted were blindfolded. The subjects were told to follow mentally a pathway moving sequentially through a series of adjacent cubes. Instructions were similar to those used by Kerr (1987); however, our instructions also included a tactual exploration of the patterns of the external and internal cubes before the start of each phase of the experiment. The subjects could use either a verbal or a tactile response, by selecting from the pattern the cube associated to the last position of the pathway.

Possible order effects were controlled, repeating the trials for each pattern in reverse order and alternating two- and three-dimensional patterns. We also adopted Kerr's (1987) practice of starting with the simplest patterns and gradually increasing the difficulty. For half the subjects, the order of presentation of the patterns was the following: $2 \times 2 \times 2,3 \times 3,3 \times 3 \times 3,4 \times 4,4 \times 4 \times 4,8 \times 8,8 \times 8$, $4 \times 4 \times 4,4 \times 4,3 \times 3 \times 3,3 \times 3$, and $2 \times 2 \times 2$. For the other half, the first pattern was the simplest two-dimensional one $(3 \times 3)$, followed by the simplest three-dimensional one, and so on.

The subjects explored each pattern tactually and received two practice trials. During the first practice trial, they were allowed to follow the pathway in the cube pattern; however, the second trial was identical to the experimental trials. If any subjects were wrong in the first practice trial, they were shown their errors, and the trial was repeated. The subjects could take a short interpattern rest. The entire session required approximately $50 \mathrm{~min}$.

\section{Results}

Mean percentages of errors for two- and three-dimensional patterns of different complexity made by the two groups of subjects appear in Figure 1. These data were analyzed with a three-way analysis of variance (ANOVA) for a complete mixed design, which used groups as a between-subject variable and the number of dimensions and the complexity of the matrices as within-subject variables.

The effects due to the number of dimensions $[F(1,38)=$ $14.47, p<.001]$, the complexity variable $[F(2,76)=$ $136.22, p<.001]$, and their interaction $[F(2,76)=6.61$, $p=.002$ ] were significant. The two groups were not significantly different $\left[F(1,38)=0.11, M S_{\mathrm{e}}=6.63, p=\right.$ $.75]$, but the group variable interacted significantly with dimensions $\left[F(1,38)=4.57, M S_{\mathrm{e}}=1.53, p=.039\right]$. A post hoc comparison using Tukey tests (both $a$ and $b$ procedures; see Winer, 1971) showed that the blind-group performance with the three-dimensional matrices was significantly lower than that of the sighted-group performance with the same matrices.

Because the data could be confounded by the presence of a few particular cases of blindness (e.g., retrolental fibroplasia and not-perfectly complete visual handicap), we excluded them (see Appendix A) and their corresponding controls from a successive similar ANOVA; however, the outcomes were exactly the same, including the interaction between groups and dimensions $\left[F(1,30)=5.36, M S_{e}=\right.$ $1.71, p=.0281$, although the interaction between dimensions and complexity was no longer significant $(p=.106)$.

Figure 1 shows that difficulty tends to increase with complexity and with a greater number of dimensions. Furthermore, an increase in the number of dimensions causes higher difficulty for the blind than for the sighted. Although the interaction between the three variables was not significant, the entries in Figure 1 suggest that this relation is most evident in the medium-complexity patterns. It is less apparent in the low-complexity patterns (where data may have been affected by a ceiling effect), and it is totally absent in the high-complexity patterns.

\section{Discussion}

Our sighted subjects differed in some respects from Kerr's (1987). In particular, the three-unit capacity limitation appears critical for two-dimensional patterns, but 


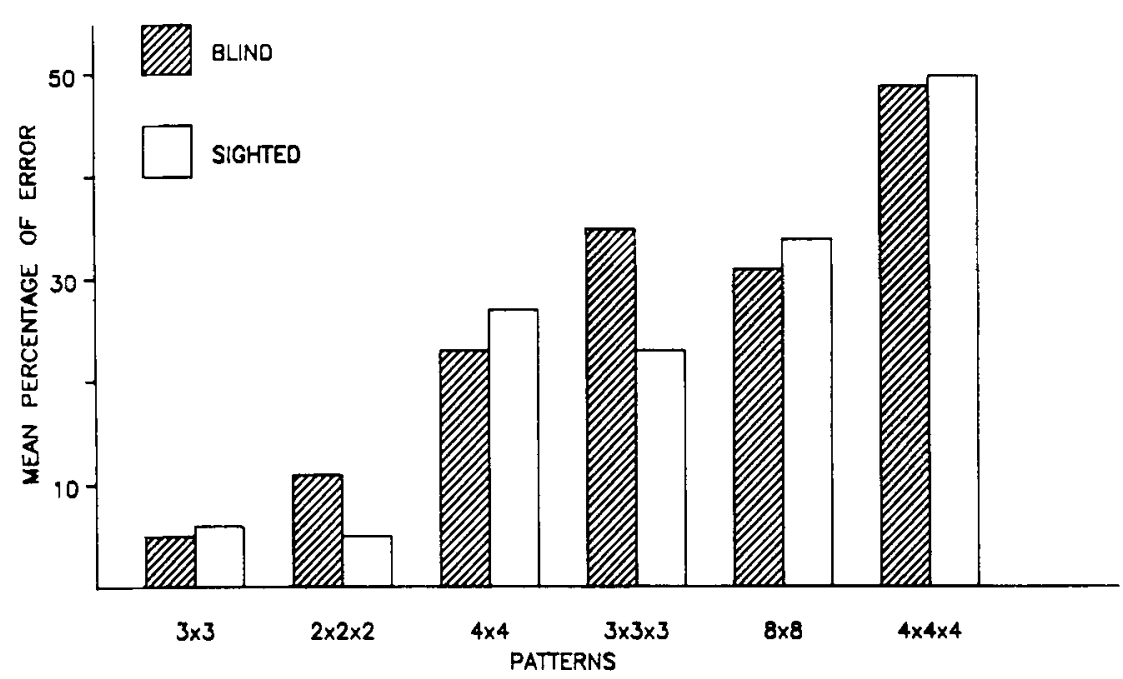

Figure 1. Mean percentages of errors for two- and three-dimensional patterns of different complexity made by a group of totally congenitally blind subjects and a matched group of sighted subjects.

not for three-dimensional patterns. The $18 \%$ difference in errors between the $2 \times 2 \times 2$ and the $3 \times 3 \times 3$ patterns is substantial and seems to show that the $3 \times 3 \times 3$ pattern involves an overload of the visuospatial sketch pad (note that, with the same presentation rate, Kerr observed only a 3\% error rate with the $3 \times 3 \times 3$ pattern in her Experiment 1 ). The $34 \%$ error rate is lower for our subjects with the $8 \times 8$ pattern than the $70 \%$ rate found by Kerr in her Experiment 3 with a 1.5 presentation rate.

The blind group's performance shows a difficulty with some three-dimensional patterns. In particular, the blind subjects had problems with the $3 \times 3 \times 3$ matrix, which seemed due to their visuospatial working-memory limitations and produced an error rate higher than that for the $8 \times 8$ matrix.

This result could be due to the fact that the blind subjects used verbal mediation instead of a visuospatial strategy. Verbal mediation might be rather ineffective with the three dimensions of the $3 \times 3 \times 3$ matrix. To examine whether the blind subjects were using verbal mediation, we tested them again.

\section{EXPERIMENT 2}

Experiment 2 was designed to compare the performance of sighted and blind groups on the visuospatial task of Experiment 1 and a heavily verbal matching task. In the verbal task, the subjects were asked to transform an object along such dimensions as sweetness and temperature. We assumed that this task would reduce the role of visuospatial processes. We also included size as a dimension because other, nonvisual, dimensions seemed to make the task abstruse to our subjects.

If the blind subjects used a verbal strategy in Experiment 1 by assigning a verbal value to each position along each dimension, their performance with the visuospatial task should be comparable to the performance they obtained with the new task, presumably requiring the use of a similar verbal strategy.

\section{Method}

Subjects. The same blind and sighted subjects who participated in Experiment 1 (except 3 sighted who were substituted by 3 other subjects with the same characteristics) participated in Experiment 2.

Materials. The material included the two patterns with three units for each side $(3 \times 3$ and $3 \times 3 \times 3)$, with the same pathways as in Experiment 1 . We prepared other series of statements that described a corresponding change of state along two dimensions (temperature and size) or three dimensions (temperature, size, and taste). For each spatial pathway, a corresponding description along these dimensions was created. For example, the spatial pathway could be "Begin with the lowest back right comer, go forward, top, left. left, backwards, top, right." The corresponding verbal pathway could start from one of the extreme cases, "bitter, small, and cold," and proceed to "bigger, sweeter, hotter, hotter, smaller, sweeter, colder."

Procedure. The procedure was the same as in Experiment 1. Order of patterns and nature of materials were balanced on the basis of two orders. The first order was $3 \times 3$, two-dimensional verbal, $3 \times 3 \times 3$, three-dimensional verbal, three-dimensional verbal, $3 \times 3 \times 3$, two-dimensional verbal, $3 \times 3$. The second order reversed the order with respect to the spatial-verbal strategy variable. During the verbal task, the sighted subjects were allowed to stay with their eyes open; during the spatial tasks, they were blindfolded.

Instructions and practice trials for the verbal tasks were comparable to those for visuospatial tasks and included a short training phase in which the subjects were asked to give answers. The subjects were tested approximately 1 month after Experiment 1.

\section{Results}

The two groups' mean percentages of errors for the different verbal and spatial patterns are shown in Figure 2. A three-way $2 \times 2 \times 2$ ANOVA on the number of errors for a mixed design revealed significant main effects due to the nature of the task (verbal vs. spatial) $[F(1,38)=$ $184.14, p<.001]$ and the number of dimensions 


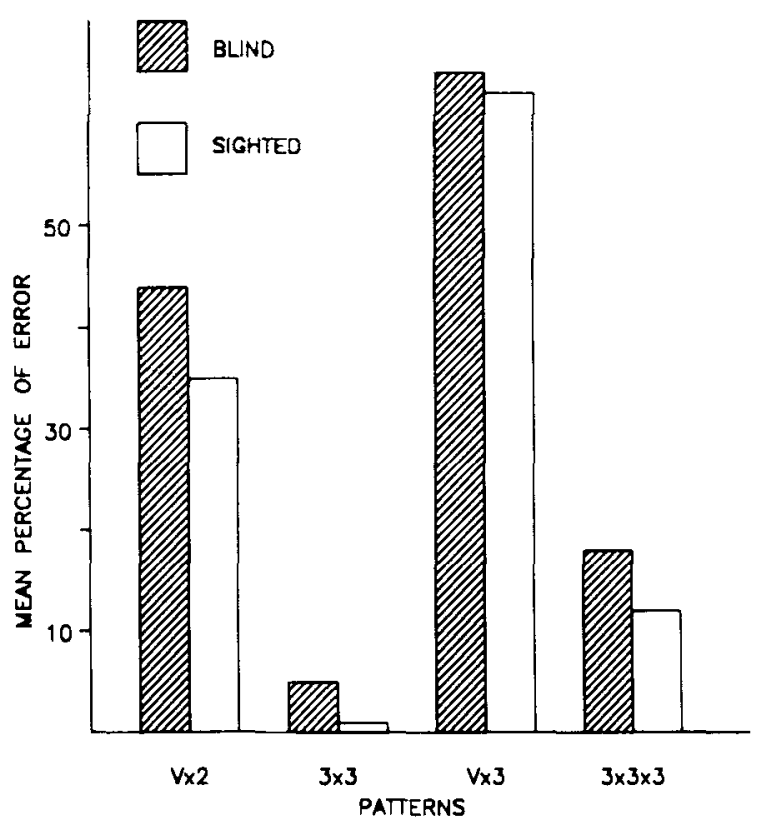

Figure 2. Mean percentages of errors for verbal two-dimensional $(V \times 2)$ or three-dimensional patterns $(V \times 3)$ and spatial twodimensional $(3 \times 3)$ and three-dimensional patterns $(3 \times 3 \times 3)$ made by the group of totally congenitally blind subjects who participated in Experiment 1 and by the matched group of sighted subjects.

$[F(1,38)=73.78, p<.001]$. Furthermore, the interaction between these two factors was significant $[F(1,38)$ $=5.95, p=.019]$. Post hoc comparisons using the Tukey test (both $a$ and $b$ procedures) revealed that errors increased with complexity for both the spatial and the verbal tasks; the increase was greater for the verbal tasks than for the spatial tasks. The difference between groups was not significant $\left[F(1,38)=1.54, M S_{e}=6.16, p=\right.$ $.22]$, nor was the interaction between groups and dimensions $\left[F(1,38)=0.13, M S_{\mathrm{e}}=1.20, p=.72\right]$.

\section{Discussion}

The absence of significant effects related to the groups shows that both groups performed the tasks in similar ways and, given the better performance on the spatial task, must have used a different, presumably visuospatial, process. Nevertheless, the data could be influenced by the fact that the subjects had already had some experience with the visuospatial task. In fact, if we compare the absolute percentages of errors for comparable patterns in the two experiments, we see that the error rate is lower for Experiment 2. For this reason, we decided to carry out a third experiment with the same procedure but with new subjects.

\section{EXPERIMENT 3}

Experiment 3 was designed to partial out a possible practice effect present in Experiment 2 by testing new groups of blind and sighted people on the same tasks.

\section{Method}

Subjects. Fifteen subjects (10 females and 5 males), classified as totally congenitally blind, aged between 15 and 58 (mean age $=24$ years, 6 months), took part in Experiment 3 . The nature of their visual handicaps is illustrated in Appendix B.

Other characteristics were similar to those of the preceding experimental subjects. Fifteen sighted controls were matched for age, sex, and school level, as in Experiments 1 and 2. The two groups were not significantly different in the performance of the WAIS digit span test (in the forward and backward digit span, the mean performances were 6.67 and 4.93 for the blind and 5.87 and 4.2 for the sighted).

Materials and Procedure. The materials and procedure were the same as in Experiment 2, except that the balancing of tasks and patterns was complete with eight different orders. The subjects could begin with either a verbal or a spatial task, either two- or threedimensional

\section{Results}

Figure 3 shows the mean percentages of errors for the two groups with different patterns and nature of material. A three-way ANOVA for a mixed design on the numbers of errors revealed the same significant main effects as in Experiment 2-that is, a main effect due to the nature of the task (spatial vs. verbal) $[F(1,28)=48.14, p<.001]$ and a main effect due to the complexity of the patterns $[F(1,28)=55.25, p<.001]$. No other significant effects were found, although we found some trends involving the groups $\left[F(1,28)=2.60, M S_{\mathrm{e}}=14.4, \mathrm{p}=.118\right]^{1}$ and the interaction among the three variables $[F(1,28)=$ $1.89, M S_{\mathrm{c}}=2.75, p=.18$ ]. Figure 3 suggests that, in

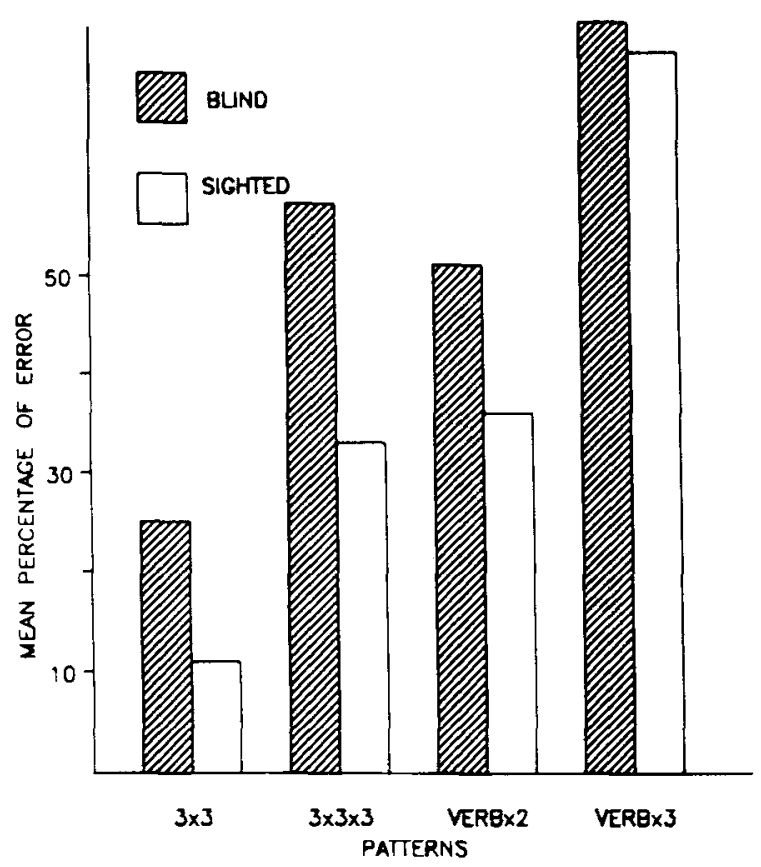

Figure 3. Mean percentages of errors for spetial two-dimensional $(3 \times 3)$ and three-dimensional patterns $(3 \times 3 \times 3)$ and verbal twodimensional $($ VERB $\times 2)$ and three-dimendonal patterns $($ VERB $\times 3$ ) made by totally congenitally blind subjects and matched sighted subjects. 
some cases, and especially with the $3 \times 3 \times 3$ matrix, the blind subjects' performance was inferior. Given these trends and our central predictions, we compared the eight mean values using the conservative Tukey $b$ post hoc test (Winer, 1971). The blind subjects' performance was significantly lower than the sighted subjects' performance on only the three-dimensional visuospatial pattern.

\section{Discussion}

The data confirm that the blind also find greater difficulty with the verbal task than with the visuospatial task and that they often respond similar to how the sighted do. Performance was lower in Experiment 3 than in Experiment 2, showing the blind's tendency to have greater difficulty with the $3 \times 3 \times 3$ spatial pattern.

The difference between the two last experiments could be due to the different level of familiarity with the spatial tasks or to different level of abilities of the subjects. The generally better performance in Experiments 1 and 2, relative to that in Experiment 3, may be due to the inclusion in Experiments 1 and 2 of the blind subjects who were cognitively more skilled (they, in fact, also had higher levels of performance in the span test). The same was also true for the sighted subjects who were matched to the blind. Given the low number of subjects with a total congenital handicap, this problem could not be avoided.

\section{EXPERIMENT 4}

In Experiment 1, the blind subjects' particular difficulty with the three-dimensional patterns was found, but only in the case of the two simpler patterns. It is possible that the most complex pattern involved a different strategy, unrelated to visuospatial processes and which the blind and sighted both used.

We reasoned that if verbal mediation is involved, a visuospatial interfering task should damage performance for only simple patterns, and a different verbal articulatory task should damage performance for only the complex patterns.

\section{Method}

Subjects. Twelve sighted subjects (6 males and 6 females), comparable for age and sociocultural characteristics to those of the preceding experiments, participated in Experiment 4.

Materials. A tape-recorded series of pathways had the same characteristics as the preceding pathways. A low-intensity filtered light torch ( $1 \mathrm{~W}, 3.8 \mathrm{~V}$ ) was used to present short flashes $(400 \mathrm{msec})$ of light, randomly at a mean frequency of 42 per minute. These flashes could be perceived only with particular attention.

Procedure. The subjects were asked to examine the patterns in the following order: $3 \times 3 \times 3$ ( 8 trials), $4 \times 4 \times 4(8+8$ trials). $3 \times 3 \times 3$ ( 8 trials). Trials were divided into blocks of four, assigned in a balanced way to either the visual or the verbal interfering condition. The first four blocks were preceded by two practice trials, as in the preceding experiments.

For the blocks with visual interference, the torch was turned on randomly and, a few seconds later, the tape-recorded pathway was played. The subject had his or her eyes open and had to press a button when the light appeared, while the pattern was covered by a screen. Immediately after the end of the pathway, the screen was climinated and the subject had 10 give the response cither verbally or by pointing to the cube.

For the blocks with verbal interference, the subjedts had to start counting in a low voice at the beginning of each trial and to continuc also while the pathway was read. The experimenter could make sure that the task was executed both by monitoring the subject's whispered counting and by noting the last number that the subject spoke out loud immediately before giving the response. The procedure was the same as in the preceding experiments in all the other respects.

\section{Results}

Mean percentages of errors for the two patterns following the two interfering tasks are presented in Figure 4. A three-way ANOVA on the number of errors for a mixed complete design was calculated. The ANOVA revealed significant main effects of the complexity of the pattern $[F(1,11)=51.79, p<.001]$ and of the nature of the interfering task $[F(1,11)=9.79, p=.01]$. Furthermore, the interaction between complexity and nature of the interfering task was significant $[F(1,11)=8.31, p=.015]$. Tukey post hoc comparisons ( $a$ and $b$ procedures) between the four mean values revealed that the difference between the two interfering conditions was significant for the $3 \times 3 \times 3$ pattern, but it was not significant for the $4 \times 4 \times 4$ pattern.

Figure 4 shows that the more complex task had a higher number of errors but was not differentially affected by the visual interfering task, unlike the simpler pattern. The result confirms our hypothesis that the absence of a difference between sighted and blind subjects with very complex patterns can be due to the fact that sighted people, too, do not use visual imagery in a specific and exclusive

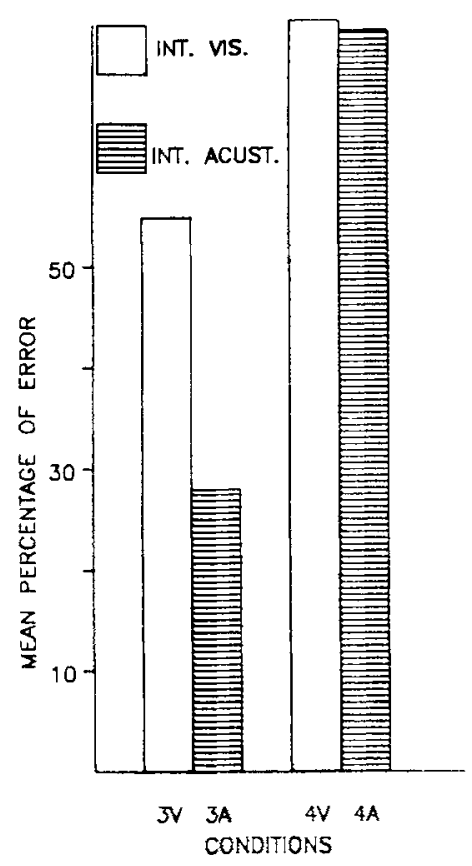

Figure 4. Mean percentages of errors for three-dimensional patterns of different complexity (three and four units per side) following different interfering conditions. 
way. It must be emphasized that the auditory task did not interfere more than did the visual task with the complex pattern; the implication of this result is that verbal mediation was also not used in an exclusive way in this case.

It also could be argued that data with the more complex matrices were the result of a ceiling effect. Although we cannot exclude the possibility that the subjects, having at least some rough idea about where the path should have ended, used some form of guessing, it must be noticed that, in general, the observed performance of approximately $70 \%$ errors is far from the performance we could expect if the subjects were simply guessing (with 64 alternatives, the chance level was greater than $98 \%$ of errors).

\section{EXPERIMENT 5}

The purpose of Experiment 5 was to see whether individual differences in the capacity of the visuospatial working memory can be found also with a group of sighted people. The large variability we had found among our subjects (e.g., Experiment 3) suggested that we examine the performance on the visuospatial tasks of groups of sighted people of varying visuospatial abilities. In particular, we wanted to see whether or not some differences in performance found between our subjects in Experiment 1 and Kerr's (1987) subjects could be explained by individual differences.

\section{Method}

Subjects. Of 32 senior high school students (19 females and 13 males, aged between 17 and 19 years), 16 belonged to a high visuospatial ability (HVS) group and 16 belonged to a low visuospatial ability (LVS) group. The subjects were part of a large sample of 123 students of the same age who were tested with the visuospatial $S$ subtest of the B.C.R. test (Reuchlin \& Valin, 1971). The subtest, which appeared capable of finding individual differences related to the visualizer-verbalizer dimension (Baroni et al., 1989), presents three-dimensional shapes from particular perspectives and requires the subjects to select, among four alternatives, the pattern that the shape should take if considered from a different predefined perspective. We assigned the 16 subjects with the highest scores $(>26)$ to the HVS group and the 16 subjects with the lowest scores $(<17)$ to the LVS group. The subjects with poor school achievement were not considered, so that the two groups were matched for school achievement as defined by the mean marks in different school areas.

Materials and Procedure. The materials and procedure were the same as in Experiment 1. The subjects were blindfolded.

\section{Results}

Mean percentages of errors are presented in Figure 5. As with Experiment 1, we carried out an ANOVA for a complete mixed design on the number of errors, using groups as a between-subjects variable and the six patterns and level of complexity (three levels) and number of dimensions (two levels) as within-subject variables. The ANOVA revealed a significant main effect of the groups $[F(1,30)=13.24, p<.01]$, a significant main effect due to the task complexity $[F(2,60)=75.58, p<.001]$, a significant group $\times$ dimension interaction $[F(1,30)=$ $14.44, p=.001]$, a significant interaction for complexity $\times$ number of dimensions $[F(2,60)=10.05$, $p<.001]$, and an interaction among all the three variables $[F(2,60)=4.92, p=.011]$. A post hoc comparison using the Tukey $b$ procedure among all the mean values showed that groups were significantly different with the $3 \times 3 \times 3$ matrix and with the $4 \times 4 \times 4$ matrix.

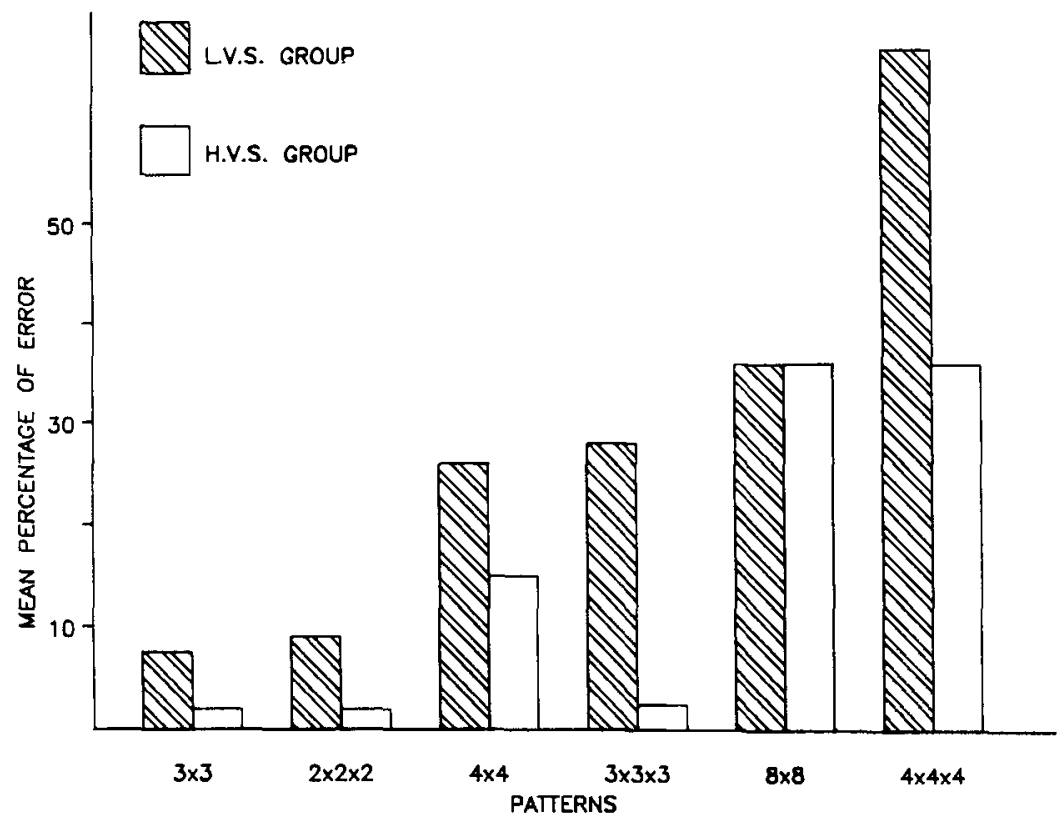

Figure 5. Mean percentages of errors for two- and three-dimensional patterns of different complexity made by a low-visuospatial-ability (LVS) group and a high-visuospatialability (HVS) group. 
As we can see from Figure 5, the HVS group tended to outperform the LVS group; the difference was particularly evident for the most difficult matrices, but not with the $8 \times 8$ pattern, and the HVS group's superiority was particularly evident with the three-dimensional complex patterns. With respect to Kerr's data, the HVS group reflects the performance of her subjects, showing an excellent performance with the $3 \times 3 \times 3$ matrix and a dramatic drop in performance with the $4 \times 4 \times 4$ matrix. On the other hand, the LVS group gave a poor performance with the $3 \times 3 \times 3$ pattern; however, the drop (unlike in our Experiment 1) was more severe between the $3 \times 3 \times 3$ pattern and the $4 \times 4 \times 4$ pattern than between the $2 \times 2 \times 2$ pattern and the $3 \times 3 \times 3$ pattem. Again, our subjects were superior to Kerr's Experiment 3 subjects with the $8 \times 8$ pattern. However, three considerations make a precise comparison difficult: (1) the fact that the rate was slower for our subjects, (2) the slight procedural differences, and (3) the fact that, in the Kerr study, the subjects of Experiment 1 were probably not comparable to the subjects of Experiment 3. Finally, the fact that a high visuospatial ability yields higher performance in the more difficult three-dimensional shapes suggests that it may be involved with a $4 \times 4 \times 4$ matrix.

\section{CONCLUSIONS}

Visuospatial working memory is a construct that, in the last few years, has been capturing more and more attention (Logie, 1990). We can expect that, as happened for the auditory component of working memory (Baddeley, 1986), particular attention will be devoted to the representational format of its content and to its capacity-two issues that concerned us. The main conclusions that can be drawn from the current research concern visuospatial imagery processes in the blind and the problem of capacity limitation in visuospatial working memory.

With respect to the first point, the data of Experiments 2 and 3 confirm that totally congenitally blind people can generate different representations by following visuospatial and verbal instructions and can profit from visuospatial representation. The blind also seem, for this task, to take a greater advantage from the visuospatial workingmemory representation than from the verbal one, probably due to the parallel properties of visual analogical representations. The fact that the blind seem able to generate such representations is not surprising when we consider that they have many opportunities, through other sensory modalities and movement, to acquire knowledge of the visuospatial properties of the world.

The current research also confirms the series of results obtained by Cornoldi and others (Cornoldi \& De Beni, 1988; Cornoldi et al., 1989; De Beni \& Cornoldi, 1988) showing that the blind may profit from imagery instructions, but within limits that are lower than those for sighted people. A plausible explanation for these limits is that only visual experience may create the ability of simultaneously managing a high number of items. Furthermore, with reference to a suggested distinction between visual and spatial processes in working memory (Baddeley, 1986), we could expect a dissociation between visual and spatial capacities in the totally congenitally blind, with the visual deficit being larger than the spatial one. The blind could be especially disadvantaged in processing visual features and contents, rather than spatial arrays that can also be experienced tactually. The results of the current research, however, showing the blind's capacity limitations with three-dimensional patterns, as well, reveal that the blind's deficit involves both visual and spatial configurations. The blind show the presence of imagery processes, but they also show a limitation in their use, relative to that of sighted people. From a neuropsychological point of view, we could speculate that, in the blind with peripheral lesions, the cortical areas involved in imagery are intact but their complete neurological maturation requires visual experience. Nevertheless, the fact that the blind seem to draw some advantage from experience with the task (Experiment 2) suggests that their deficit is due to limited experience and practice with three-dimensional shapes.

With respect to the second issue, the results of Experiments 1 and 5 show that individual differences may contribute to both quantitative and qualitative differences in visuospatial working-memory capacity. In fact, the subjects with a lower capacity not only showed a lower performance, they also had particular difficulty with the three-dimensional $3 \times 3 \times 3$ matrix. This matrix appears within the capacity limitations of high-visuospatial-ability subjects. The fact that this difference disappeared (Experiment 1) with the most complex matrices is not surprising, because, in this case, specific visuospatial processes were not the only ones involved (Experiment 4). Further research is necessary to explore the implications of a third dimension for visuospatial imagery and the reasons only some subjects are not overloaded when it is involved. At the same time, further exploration of the boundary conditions related to the procedure will help to clarify the differences. For example, if we consider some differences between our results and those of Kerr (1987), we cannot decide whether they were due to some differences in the procedure (and, in particular, to the fact that our subjects, being blindfolded, had to give a tactual response rather than simply pointing to the correct cube) or to differences in ability.

\section{REFERENCES}

Attneave, F., Curlee, T. E. (1983). Locational representation in imagery: A moving spot task. Journal of Experimental Psychology: Human Perception \& Performance, 9, 20-30.

BadDELEY, A. D. (1986). Working memory. Oxford: Oxford University Press.

Baroni, M. R., Cornoldi, C., De Beni, R., D'Urso, V., Mainardi Peron, E., Palomba, D., Stegagno, L. (1989). Emozioni in celluloide. Milano: Cortina.

CoRNOLDI, C., DE BENI, R. (1988). Weaknesses of imagery without visual experience: The case of the total congenital blind using imaginal mnemonics. In M. Denis, J. Engelkamp, \& J. T. E. Richardson (Eds ), Cognitive and neuropsychological approaches to mental imagery (pp. 393-401). Dordrecht: Martinus Nijhoff. 
Cornoldi, C., De Beni, R., Roncar, S., Romano, S. (1989). The effects of imagery instructions on total congenital blind recall. European Journal of Cognitive Psychology, 1, 321-331.

Craig, E. M. (1973). Role of mental imagery in free recall of deaf, blind, and normal subjects. Journal of Experimental Psychology, 97 , 249-253.

DE Bent, R., Cornold, C. (1988). Imagery limitations in totally congenitally blind subjects. Joumal of Experimental Psychology: Learning, Memory, \& Cognition, 14, 650-655.

JoNIDEs, J., KAHN, R., RozIN, P. (1975). Imagery instructions improve memory in blind subjects. Bulletin of the Psychonomics Society, 5, 424-426.

KeEnan, J. M., Moore, R. E. (1979). Memory for images of concealed objects: A reexamination of Neisser and Kerr. Journal of Experimental Psychology: Human Leaming \& Memory, 5, 374-385.

KERR, N. H. (1983). The role of vision in "visual imagery" experiments: Evidence from the congenitally blind. Journal of Experimental Psychology: General, 112, 265-277.

KERR, N. H. (1987). Locational representation in imagery: The third dimension. Memory \& Cognition, 15, 521-530.

Kosslyn, S. M. (1980). Image and mind. Cambridge, MA: Harvard University Press.

LoGIE, R. H. (1989). Characteristics of visual short-term memory. European Journal of Cognitive Psychology, 1, 275-284.

LoGIE, R. H. (1990). Qualities of the image and the visual buffer. In C. Cornoldi \& M. McDaniel (Eds.), Imagery and cognition (pp. 77. 102). New York: Springer.

MARMOR, G. S., ZABACK, L. A. (1976). Mental rotation by the blind: Does mental rotation depend on visual imagery? Joumal of Experimental Psychology: Human Perception \& Performance, 2. 515-521.
MILLAR, S. (1975). Spatial memory by blind and sighted children. British Journal of Psychology, 66, 449-459.

Neisser, U., \& KerR, N. (1973). Spatial and mnemonic properties of visual images. Cognitive Psychology, 5, 138-150.

Phillips, W. A., Christie, D. F. M. (1977). Components of visual memory. Quarterly Journal of Experimental Psychology, 29. $117-133$

Reuchlin, M., Valin, E. (1971). Test B.C.R. Paris: Service de Recherches de l'INOP. (Italian adaptation, Firenze: O.S.)

Shagan, J., GoOdNow, J. (1973). Recall of haptic information by blind and sighted individuals. Joumal of Experimental Psychology, 101, 221-226

WINER, B. J. (1971). Statistical principles in experimental design (2nd ed.). New York: McGraw-Hill.

ZiMLER, J., KEENAN, J. M. (1983). Imagery in the congenitally blind: How visual are visual images? Journal of Experimental Psychology: Leaming, Memory, \& Cognition, 9, 269-282.

\section{NOTE}

1. Since the two groups were slightly different in the digit-memory tests, we repeated the analysis of variance, using the span scores as covariates. The blind group's performance was significantly poorer both when the covariate was the backward digit span $[F=5.78, p=.023]$ and when the covariate was the summed digit span (forwards + backwards digit span; $p=.049$ ). In all other respects, the covariance anaysis replicated the preceding effects. We also repeated the analysis by excluding the 6 atypical blind subjects, but, again, we replicated the preceding results.

APPENDIX A

Sample Descriptions of Subjects in Experiment 1

\begin{tabular}{|c|c|c|c|c|}
\hline $\begin{array}{l}\text { Subject } \\
\text { Number }\end{array}$ & Occupation & Sex & Age & Etiology and Characteristics of the Visual Handicap \\
\hline 1 & Student & $\mathrm{F}$ & 15 & Total blindness, due to virus, since lst month of life \\
\hline 2 & Student & $\mathrm{M}$ & 15 & Congenital handicap due to pigmentary retinis-only light perception \\
\hline $3 *$ & Student & $\mathrm{F}$ & 15 & $\begin{array}{l}\text { Total blindness in connection with retrolental fibroplasia due to } \\
\text { incubator oxygen }\end{array}$ \\
\hline $4^{*}$ & Student & $\mathrm{F}$ & 17 & $\begin{array}{l}\text { Total blindness in connection with retrolental fibroplasia due to } \\
\text { incubator oxygen }\end{array}$ \\
\hline 5 & Student & $\mathrm{F}$ & 17 & $\begin{array}{l}\text { Visual handicap, due to pigmentary retinis, since the } 4 \text { th month } \\
\text { of life-only shadow perception }\end{array}$ \\
\hline 6 & $\begin{array}{l}\text { Unemployed } \\
\text { (elementary teacher diploma) }\end{array}$ & $\mathrm{F}$ & 20 & $\begin{array}{l}\text { Visual handicap, due to virus, since the } 4 \text { th month of life }- \text { only } \\
\text { shadow perception }\end{array}$ \\
\hline $7^{*}$ & $\begin{array}{l}\text { Operator } \\
\text { (professional school diploma) }\end{array}$ & $\mathrm{F}$ & 27 & $\begin{array}{l}\text { Total blindness in connection with retrolental fibroplasia due to } \\
\text { incubator oxygen }\end{array}$ \\
\hline 8 & Student & M & 27 & Total blindness, due to pigmentary retinis, since birth \\
\hline 9* & Student & $\mathrm{F}$ & 27. & Congenital blindness (glaucoma), became total at the age of 4 \\
\hline 10 & Student & $\mathrm{F}$ & 28 & $\begin{array}{l}\text { Visual handicap, due to pigmentary retinis, since the Ist month } \\
\text { of life-only light perception }\end{array}$ \\
\hline 11 & $\begin{array}{l}\text { Operator } \\
\text { (professional diploma) }\end{array}$ & $\mathrm{F}$ & 35 & Total congenital blindness due to pigmentary retinis \\
\hline 12 & $\begin{array}{l}\text { Computer operator } \\
\text { (master's degree) }\end{array}$ & M & 35 & Visual handicap due to pigmentary retinis-large-forms perception \\
\hline 13 & $\begin{array}{l}\text { Operator } \\
\text { (professional diploma) }\end{array}$ & $\mathrm{F}$ & 37 & Total congenital blindness due to mother's rubella \\
\hline 14 & $\begin{array}{l}\text { Operator } \\
\text { (professional diploma) }\end{array}$ & $\mathrm{F}$ & 40 & Congenital glaucoma-only shadow perception \\
\hline 15 & $\begin{array}{l}\text { Office worker } \\
\text { (master's degree) }\end{array}$ & M & 41 & Total congenital blindness due to hereditary pigmentary retinis \\
\hline 16 & $\begin{array}{l}\text { Operator } \\
\text { (professional diploma) }\end{array}$ & M & 42 & $\begin{array}{l}\text { Visual handicap, since the } 4 \text { th month of life, due to meningitis- } \\
\text { only light perception }\end{array}$ \\
\hline 17 & $\begin{array}{l}\text { Masseur } \\
\text { (senior high school diploma) }\end{array}$ & M & 48 & $\begin{array}{l}\text { Visual handicap, since the 6th month of life, due to meningitis- } \\
\text { only light perception }\end{array}$ \\
\hline
\end{tabular}


APPENDIX A (continued)

\begin{tabular}{clcccc}
\hline $\begin{array}{c}\text { Subject } \\
\text { Number }\end{array}$ & Occupation & Sex & Age & & Etiology and Characteristics of the Visual Handicap \\
\hline 18 & $\begin{array}{l}\text { Operator } \\
\text { (professional diploma) }\end{array}$ & F & 54 & Total congenital blindness due to pigmentary retinis \\
19 & $\begin{array}{l}\text { Unemployed } \\
\text { (senior high school diploma) } \\
\text { Masseuse } \\
\text { (junior high school diploma) }\end{array}$ & F & 59 & 60 & $\begin{array}{l}\text { Total blindness, since the 1st year of life, due to pigmentary retinis } \\
\text { Congenital blindness due to pigmentary retinis-only light } \\
\text { perception }\end{array}$ \\
\hline
\end{tabular}

*Subject was excluded in final ANOVA mentioned in the Results section of Experiment 1.

APPENDIX B

Sample Descriptions of Subjects in Experiment 3

\begin{tabular}{|c|c|c|c|c|}
\hline $\begin{array}{l}\text { Subject } \\
\text { Number }\end{array}$ & Occupation & Sex & Age & Etiology and Characteristics of the Visual Handicap \\
\hline 1 & Student & $F$ & 15 & Total congenital blindness (glaucoma) \\
\hline 2 & Student & $\mathbf{F}$ & 15 & Total congenital blindness (glaucoma) \\
\hline 3 & Student & M & 15 & Total blindness since the 2 nd year of life \\
\hline 4 & Student & $\mathbf{F}$ & 17 & Total blindness \\
\hline 5 & Student & M & 17 & $\begin{array}{l}\text { Congenital blindness (glaucoma), became total at the age of } 4 \text {-only } \\
\text { light perception }\end{array}$ \\
\hline 6 & $\begin{array}{l}\text { Operator } \\
\text { (professional school diploma) }\end{array}$ & $\mathrm{F}$ & 18 & Total congenital blindness (tumor) \\
\hline 7 & Student & $\mathbf{M}$ & 18 & $\begin{array}{l}\text { Congenital blindness due to lesions to retinal cells-only light } \\
\text { perception }\end{array}$ \\
\hline 8 & Student & $\mathrm{F}$ & 19 & Total blindness \\
\hline 9 & Student & M & 19 & Congenital blindness (glaucoma)-only light perception \\
\hline 10 & Student & $\mathrm{F}$ & 20 & Total congenital blindness in relationship with mother's toxoplasmosis \\
\hline 11 & $\begin{array}{l}\text { Operator } \\
\text { (senior high school diploma) }\end{array}$ & $\mathrm{F}$ & 24 & Congenital optical nerve athrophy-only light perception \\
\hline 12 & Student & M & 28 & Congenital optical nerve athrophy-only light perception \\
\hline 13 & Operator & $\mathrm{F}$ & 40 & $\begin{array}{l}\text { Congenital blindness in relationship with pigmentary retinis-only } \\
\text { light perception }\end{array}$ \\
\hline 14 & $\begin{array}{l}\text { Masseuse } \\
\text { (professional school diploma) }\end{array}$ & $\mathrm{F}$ & 45 & Total blindness, due to a virus, since the 2 nd year of life \\
\hline 15 & $\begin{array}{l}\text { Unemployed } \\
\text { (senior high school diploma) }\end{array}$ & $\mathbf{F}$ & 58 & Blindness, following meningitis, since the $3 \mathrm{rd}$ month of life \\
\hline
\end{tabular}

(Manuscript received April 20, 1990;

revision accepted for publication February 14, 1991.) 